\title{
A scheduling method to reduce waiting time for close-range broadcasting
}

\author{
Yusuke Gotoh $^{\mathrm{a}, \mathrm{b}, *}$, Tomoki Yoshihisa ${ }^{\mathrm{c}}$, Hideo Taniguchi ${ }^{\mathrm{a}}$, Masanori Kanazawa ${ }^{\mathrm{d}}$, \\ Wenny Rahayu ${ }^{\mathrm{b}}$ and Yi-Ping Phoebe Chen ${ }^{\mathrm{b}}$ \\ ${ }^{a}$ Okayama University, Okayama, Japan \\ ${ }^{\mathrm{b}}$ La Trobe University, Melbourne, VIC, Australia \\ ${ }^{\mathrm{c}}$ Osaka University, Osaka, Japan \\ ${ }^{\mathrm{d}}$ The Kyoto College of Graduate, Studies for Informatics, Kyoto, Japan
}

\begin{abstract}
Due to the recent popularization of digital broadcasting systems, close-range broadcasting using continuous media data, i.e. audio and video, has attracted great attention. For example, in a drama, after a user watches interesting content such as a highlight scene, he/she will watch the main program continuously. In close-range broadcasting, the necessary bandwidth for continuously playing the two types of data increases. Conventional methods reduce the necessary bandwidth by producing an effective broadcast schedule for continuous media data. However, these methods do not consider the broadcast schedule for two types of continuous media data. When the server schedules two types of continuous media data, waiting time that occurs from finishing the highlight scene to starting the main scene, may increase. In this paper, we propose a scheduling method to reduce the waiting time for close-range broadcasting. In our proposed method, by dividing two types of data and producing an effective broadcast schedule considering the available bandwidth, we can reduce the waiting time.
\end{abstract}

Keywords: Close-range broadcasting, scheduling, waiting time, continuous media data

\section{Introduction}

Due to the recent popularization of digital broadcasting systems, close-range broadcasting using continuous media data, i.e. audio and video, has attracted great attention. Examples follow:

- In news programs, after watching a news headline, the user watches the main news continuously.

- In dramas, the user first watches a highlight scene. If the user has an interest in this drama, he/she watches the main program.

In our paper, we consider the case of delivering data for close-range broadcasting. In broadcasting systems, clients generally have to wait until their desired data is broadcasted. Therefore, there is many research on reducing the waiting time. We have proposed several scheduling methods to reduce the waiting time without interruption. These methods reduce the waiting time before playing data. However, we did not consider the case of close-range broadcasting. In close-range broadcasting, the server needs to deliver two types of continuous media data: one is the highlight scene and the other is the main program. When the client watches the highlight scene and the main program sequentially, in

\footnotetext{
${ }^{*}$ Corresponding author: Yusuke Gotoh, Okayama University, Okayama, Japan. E-mail: gotoh@ @s.okayama-u.ac.jp.
} 


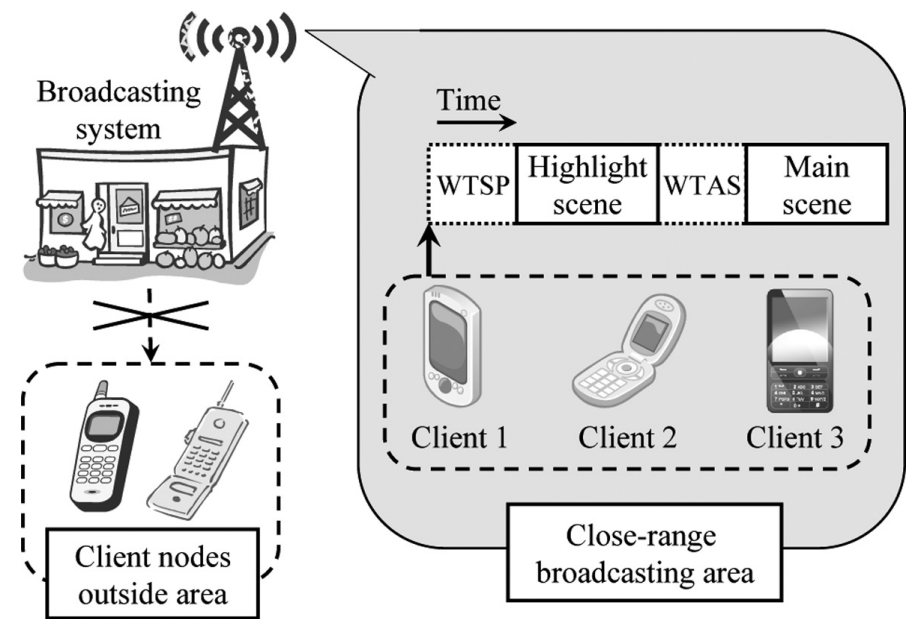

Fig. 1. Assumed structure of close-range broadcasting.

addition to the waiting time before starting the highlight scene, waiting time occurs between finishing the highlight scene and starting the main program. By increasing the available bandwidth for delivering it, waiting time before starting the highlight scene can be reduced. On the other hand, since the available bandwidth for delivering the main program becomes relatively short, the waiting time between finishing the highlight scene and starting the main program increases significantly and the total waiting time may increase.

In this paper, we propose a scheduling method to reduce the waiting time in close-range broadcasting. Our scheduling method considers the waiting time between finishing the highlight scene and starting the main program. Also, the total waiting time is reduced by calculating the available bandwidth of each channel considering the playing time of the highlight scene and the main program and making the broadcast schedule.

The remainder of this paper is organized as follows. We explain the research interest in close-range broadcasting in Section 2. Related works are introduced in Section 3. Our proposed method is explained in Section 4 and evaluated in Section 5. Finally, we conclude the paper in Section 6.

\section{Close-range broadcasting}

\subsection{Basic idea}

In this section, we explain close-range broadcasting. The assumed structure of close-range broadcasting is shown in Fig. 1. In close-range broadcasting, interference between channels can be reduced by controlling the output of radio waves and limiting the range for receiving data. The server can deliver continuous media data such as audio and video in a limited area. Users can improve convenience by receiving information according to the watching area using their nodes. Examples of such situations follow:

- In sport events, users can acquire the information of their favorite team and the situation of the game in real time.

- In the city, users can get the coupon at their favorite shops and watch the highlight scene of their interested movie. 


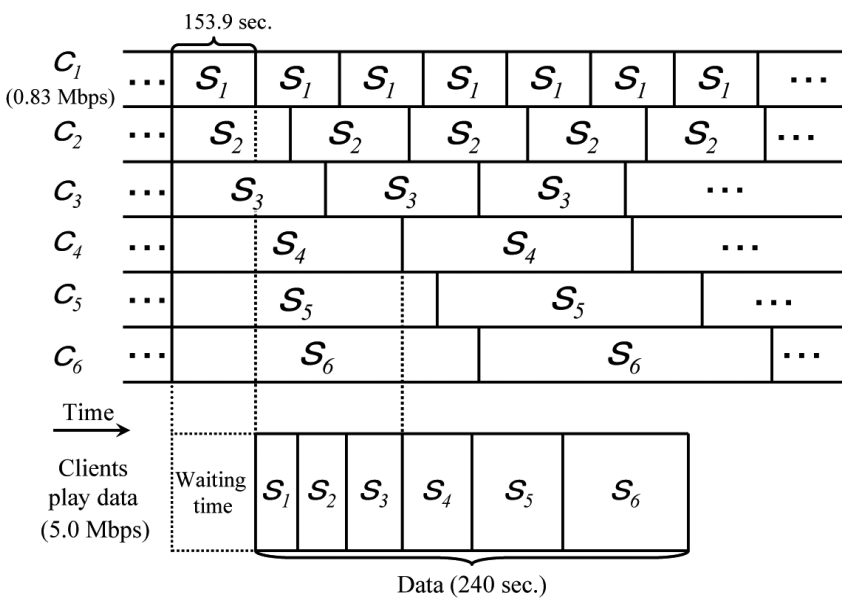

Fig. 2. Example of division-based broadcasting under BE-AHB method.

The server can deliver a program and advertisements effectively to many clients in a limited area, and clients can watch the program using their devices. However, since users move frequently, they may interrupt the program by moving out of the broadcast area. Therefore, to maintain a user's interest and encourage him/her to stay as long as possible in the area, the server needs to deliver two types of continuous media data: the highlight scene and the main program. Users first watch the highlight scene in the close-range broadcasting area. If the users are interested in the highlight scene, they would watch the main program continuously.

In close-range broadcasting, there are two types of waiting time. We call the waiting time that occurs from selecting the highlight scene to starting to play "waiting time after selection" (WTAS). The server can set an upper limit for WTAS. Also, we call the waiting time that occurs from requiring the program to starting to play "a waiting time for starting the program" (WTSP). WTAS occurs when requiring a user's selection. On the other hand, WTSP occurs when starting to play the data.

If the waiting time occurs after starting to watch the main program, continuity of the program is interrupted and users feel annoyed. Conventional methods do not distinguish WTAS and WTSP. In this paper, we reduce the waiting time effectively by making the broadcast schedule considering WTAS. The duration of the acceptable waiting time depends on the users, but we suppose that acceptable waiting time is given considering audience rating and so on.

\subsection{Mechanism for waiting time}

In this subsection, we explain the mechanism for waiting time generation. When the server broadcasts continuous media data repetitively, clients have to wait until the first portion of the data is broadcasted. For example, when the server broadcasts MPEG2-encoded music clip data whose receiving time is $60 \mathrm{~min}$, waiting time is $60 \mathrm{~min}$. To reduce the waiting time, many methods employ the division-based broadcasting technique, which reduces the waiting time by dividing the data into several segments and broadcasting precedent segments frequently. In division-based broadcasting, these methods suppose data delivery such as radio wave broadcasting and Internet multicast.

In division-based broadcasting, since the data are divided into several segments, segments must be scheduled without interrupting the clients' continuous play. In the conventional methods, clients can play data without interruption considering available bandwidth and several channels. 


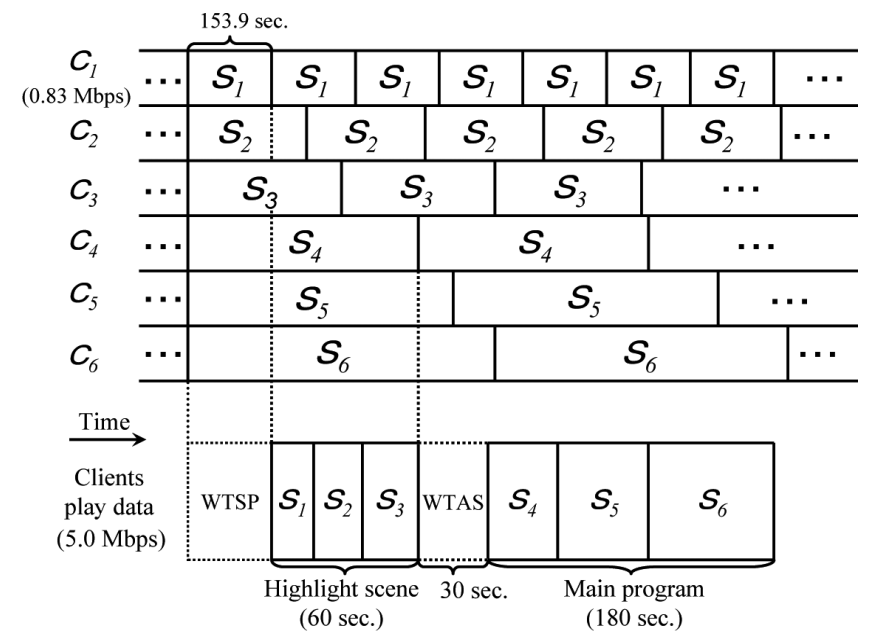

Fig. 3. Example of close-range broadcasting under BE-AHB method.

An example of division-based broadcasting is shown in Fig. 2. The example uses the Bandwidth Equivalent-Asynchronous Harmonic Broadcasting (BE-AHB) method [15] to explain the problem of waiting time. This example divides data into six segments relatively. The playing time is $240 \mathrm{sec}$. The data is divided into six segments, $S_{i}(i=1, \cdots, 6)$. When the available bandwidth for clients is $5.0 \mathrm{Mbps}$, the bandwidth of each channel is $5.0 / 6=0.83$ Mbps. The consumption rate is $5.0 \mathrm{Mbps}$. Under the BE-AHB method, when the total playing time is $60+180=240 \mathrm{sec}$, the playing time of $S_{1}$ is $25.5 \mathrm{sec}$, $S_{2}$ is $29.8 \mathrm{sec}, \cdots$, and $S_{6}$ is $55.3 \mathrm{sec}$. The server repetitively broadcasts $S_{i}$ by broadcast channels $C_{i}$. Clients can play each segment after receiving it. While playing the data, clients receive the broadcast data and store it in their buffers. In this case, clients can play the data continuously until it has ended, even if they start playing it immediately after completely receiving $S_{1}$. When clients finish playing $S_{1}$, they have finished receiving $S_{2}$ and can play $S_{2}$ continuously. Also, when they are finished playing $S_{3}$, they have finished receiving $S_{4}$ and can play $S_{4}$ continuously. In this case, waiting time is $25.5 \times$ $5.0 / 0.83=153.9 \mathrm{sec}$, which is the same as the time needed to receive only $S_{1}$. In the simple method, since the server broadcasts data without dividing it, waiting time is $240 \times 5.0 / 5.0=240 \mathrm{sec}$. Therefore, $(240-153.9) / 240 \times 100=35.9 \%$, which is shorter than the simple method.

Next, in close-range broadcasting, the broadcast schedule under the BE-AHB method is shown in Fig. 3. We set WTAS to be $30 \mathrm{sec}$. In Fig. 3, the server broadcasts partitioned data repetitively under the BE-AHB method using both the highlight scene and main program. In close-range broadcasting, WTAS occurs while playing the program. On the other hand, in the BE-AHB method, since clients play segments without interruption, the BE-AHB method does not make the broadcast schedule considering WTAS and cannot reduce waiting time effectively.

\section{Related works}

In Japan, several services using close-range broadcasting have been proposed. Area One segmentBroadcasting (Area One-Seg) [2] is a broadcasting service that delivers data by applying one-segment technology in a limited area within a one-kilometer radius. One-segment technology is used for terrestrial digital broadcasting in Japan. By delivering content suitable for the place and time, the server can 
deliver information effectively in a gathering place such as a station or a stadium. Spot one-segment broadcasting [3] is a service in which the server can broadcast data to specific users. In spot one-segment broadcasting, everybody can manage the broadcast service without a broadcast license. However, electric waves in one-segment broadcasting are weaker than in Area One-Seg, and the range of transmission is limited to about a one-meter radius. In these broadcasting systems, the broadcast scheduling has not been proposed to reduce waiting time.

Several methods to reduce waiting time have been proposed in continuous media data broadcasting $[4$, 6-9]. These methods reduce waiting time by dividing the data into several segments and producing an efficient broadcast schedule. In Heterogeneous Receiver-Oriented Broadcasting (HeRO) [12], the data are divided into different sizes. Let $J$ be the data size for the first segment. The data sizes for the segments are $J, 2 J, 2^{2} J, \ldots, 2^{K-1} J$. However, since the data size of the $K^{t h}$ channel becomes half of the data, clients may experience waiting time and interruptions.

In BroadCatch [13], the server divides the data into $2^{K-1}$ segments of equal size and broadcasts them periodically using $K$ channels. The bandwidth for each channel is the same as the data bit rate. By adjusting $K$ based on the available bandwidth for clients, waiting time is effectively reduced. However, since the available bandwidth is proportional to the number of channels, when an upper limit exists in the server's bandwidth, the server might not be able to acquire enough channels to broadcast the data.

In Harmonic Broadcasting (HB) [14], the data is separated into $S_{1}, \cdots, S_{N}$ of equal size. The server sets $C_{1}, \cdots, C_{N}$ channels considering the available bandwidth and schedules $S_{1}, \cdots, S_{N}$. The client can play $S_{1}, \cdots, S_{N}$ without interruption using $C_{1}, \cdots, C_{N}$. For example, when the server broadcasts continuous media data whose playing time is $60 \mathrm{~min}$ and whose consumption rate is 5.0 Mbps using 24 Mbps, which is identical to that under digital broadcasting systems, we need 67 channels.

In Asynchronous Harmonic Broadcasting (AHB) [15], waiting time is reduced more than the HB method by scheduling the playing unit time, such as Group of Pictures (GOP) in MPEG2 or the frame in MP3. Since the server divides the data of each playing unit time, the number of channels is the same as the number of playing units. For example, when the server broadcasts a piece of continuous media data whose playing time is $60 \mathrm{~min}$ and whose consumption rate is 5.0 Mbps using $24 \mathrm{Mbps}$, which is identical to that under digital broadcasting systems, the number of channels is 6,000 . The BE-AHB method is an extended version of the AHB method, where continuous media data is separated by a fixed data size. The server can reduce the necessary number of channels, which is more realistic than the original AHB method.

Kua et al. proposed an algorithm for answering spatial nearest neighbor search queries by leveraging results from neighboring nodes within a mobile environment [16]. This algorithm allows a mobile node to locally verify whether candidate objects received from the neighbors are indeed part of its own nearest neighbor data set.

We previously proposed scheduling methods to reduce waiting time for division-based broadcasting $[17,18]$. These methods make the schedule using single continuous media data. In addition, our assumed structure is close-range broadcasting.

\section{Proposed method}

\subsection{Basic idea}

We propose a scheduling method in close-range broadcasting called the "Close-Range Harmonic Broadcasting (CR-HB)" method. We previously proposed the BE-AHB method [15]. The BE-AHB 
Table 1

Formulation symbols

\begin{tabular}{cl}
\hline Sign & Explanation \\
\hline$r$ & Consumption rate \\
$N$ & Number of segments \\
$S_{1}$ & Data of highlight scene \\
$S_{2}$ & Data of main program \\
$s_{i-j}$ & $j^{t h}$ segment in $S_{i}, i=1,2 \| j=1, \cdots, N$ \\
$C_{i}$ & Available bandwidth to broadcast $S_{i}$ \\
$c_{i-j}$ & Available bandwidth to broadcast $s_{i-j}$ \\
$T_{i}$ & Playing time of $S_{i}$ \\
$t_{i-j}$ & Playing time of $s_{i-j}$ \\
$B$ & Total available bandwidth of server \\
$p_{i-j}$ & Ratio of playing time \\
$\delta$ & Maximum WTAS \\
\hline
\end{tabular}

method reduces waiting time based on the idea explained in Section 2.2. The main difference between the CR-HB and the BE-AHB methods is considering WTAS, which occurs between finishing the highlight scene and starting the main program.

\subsection{Assumed environment}

Our assumed system environment is listed below.

- Clients play the highlight scene and the main program sequentially.

- The waiting time occurs when starting to play the highlight scene and the main program.

- Bandwidth is stable while broadcasting the data.

- Clients can start playing a segment after they have completely received it.

- The server broadcasts segments repetitively using multiple channels.

- Once clients start playing the data, they can play it without interruption.

- Clients have adequate buffer to store the received data.

\subsection{Modeling to reduce waiting time}

We developed an expression to reduce waiting time for continuous media data in close-range broadcasting. The formulation values are summarized in Table 1.

In continuous media data broadcasting, we need to schedule segments based on a receiving time of $s_{1-1}$ and interruption time between finishing the highlight scene and starting the main program. Actually, many network structures exist for division-based broadcasting systems. However, since the number of patterns is excessive, evaluating the performance of our proposed method for all of them is not realistic. Therefore, in this paper, we use the network configuration shown in Fig. 1. Although the practical programs do not always match these patterns, there are enough to show the effectiveness of our proposed method.

In our proposed method, the server reduces waiting time by calculating the available bandwidth of each broadcast channel to minimize the average waiting time. By including WTAS in the receiving time of the data, the server can lengthen the receiving time of the main program and reduce the necessary bandwidth for delivering the highlight scene. By considering WTAS, calculating the available bandwidth of each channel becomes slightly more complex. However, the available bandwidth of each channel can be calculated by a simple simulation. 


\subsection{Scheduling process}

In this section, we explain the CR-HB method. In the CR-HB method, the total available bandwidth of the server is divided into several channels. Clients can reduce waiting time effectively by scheduling segments using several channels.

The scheduling process continues as follows. Notation is defined in Table 1. We explained the basic idea of our scheduling process in Subsection 4.3.

1. According to $N, S_{i}$ separates into $s_{i-1}, \cdots, s_{i-N}$, for which the playing time is $t_{i-1}, \cdots, t_{i-N}$.

2. $s_{1-1}, \cdots, s_{1-N}$ must be scheduled so that clients that can concurrently receive data from $N$ channels, can play the data continuously. Since the playing time of $s_{1-1}$ is $\frac{C_{1}}{N r}, s_{1-2}$ must play until finishing to receive $s_{1-1}$, which is $\left(1+\frac{C_{1}}{N r}\right)$. Next, clients can wait starting of $s_{2-1}$ until finishing time of WTAS. Also, $p_{2-j}$ is calculated considering $p_{2-1}$. Therefore, when $p_{1-1}$ is $1.0, p_{i-j}$ can obtain the following equation.

$$
p_{i-j}=\left\{\begin{array}{l}
\left(1+\frac{C_{1}}{N r}\right)^{j-1}(i=1,2 \leqslant j \leqslant N) . \\
\frac{B-C_{1}}{N r}\left\{\left(1+\frac{N r}{C_{1}}\right) \times t_{1-N}+\delta\right\} .(i=2, j=1) . \\
\left(1+\frac{B-C_{1}}{N r}\right)^{j-1} \times t_{2-1}(i=2,2 \leqslant j \leqslant N),
\end{array}\right.
$$

3. $t_{i-j}$ is calculated as follows:

$$
t_{i-j}=T_{i} \times \frac{p_{i-j}}{\sum_{j=1}^{N} p_{i-j}} .
$$

4. Decide $C_{i}$ based on $t_{i-j}$ calculated in step 3. As shown in Subsection 4.5, we can calculate $C_{i}$ in the simple computational simulation.

\subsection{A simple example to reduce waiting time}

In the CR-HB method, when the available bandwidth of highlight scene is $C_{1}$, the broadcast is scheduled so that clients that can receive data from $C_{1}$ can play the data without interruption even if they start playing the data just after receiving the first segment $S_{1}$. The waiting time for clients that receive data using larger bandwidth than $C_{1}$ cannot be improved. Let $C_{1}^{*}$ be the value of optimal $C_{1}$. Then, we have to find the value of $C_{1}^{*}$ that makes the average waiting time minimum.

When $C_{1}$ is the same as the available bandwidth given by the CR-HB method, it becomes optimum. This is because the broadcast schedule set up in order to receive the next segment just before the client finishes playing the current segment. Otherwise, the broadcast schedule is not optimal. If we call the state that there is no buffer in the meantime as a starvation, we have to make the broadcast schedule so that there is no starvation as much as possible. The deadline means the time to finish playing the current segment.

Here, the procedure for calculating $C_{1}^{*}$ continues as follows:

1. Produce the broadcast schedule for all $C_{1}$.

2. Calculate the waiting time for all produced broadcast schedules.

3. Find $C_{1}^{*}$ that gives the minimum average waiting time. 


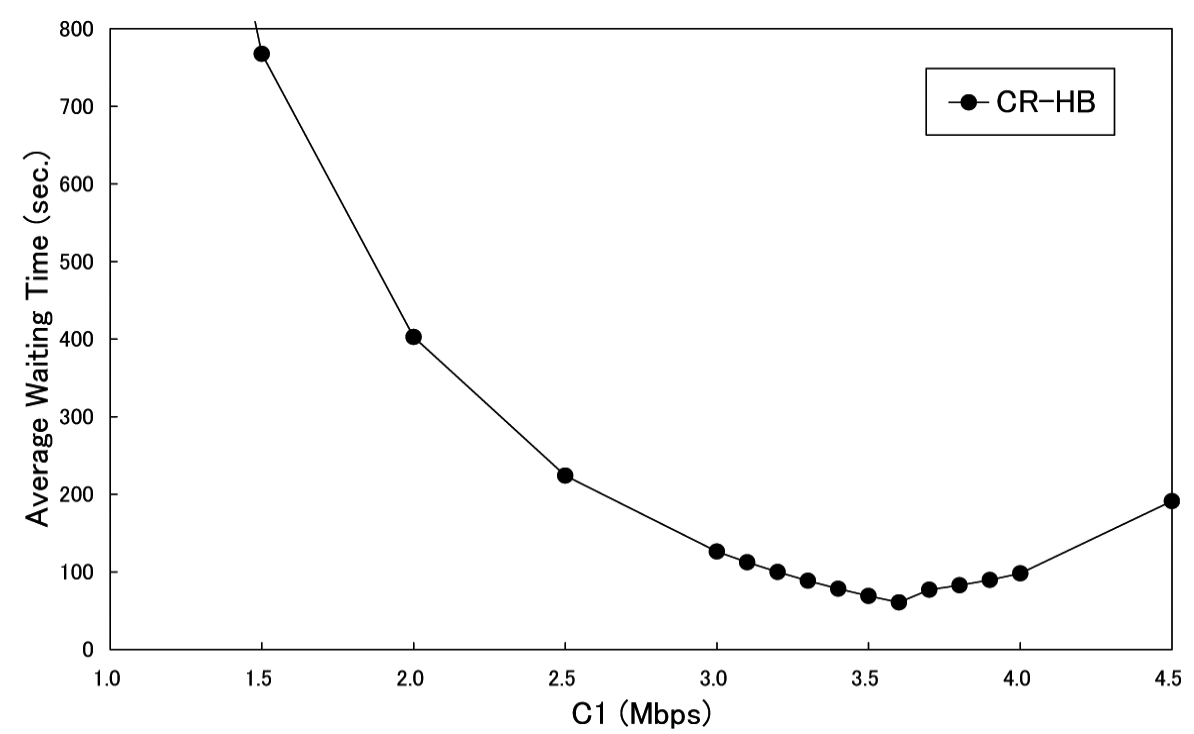

Fig. 4. Average waiting time and $C_{1}$.

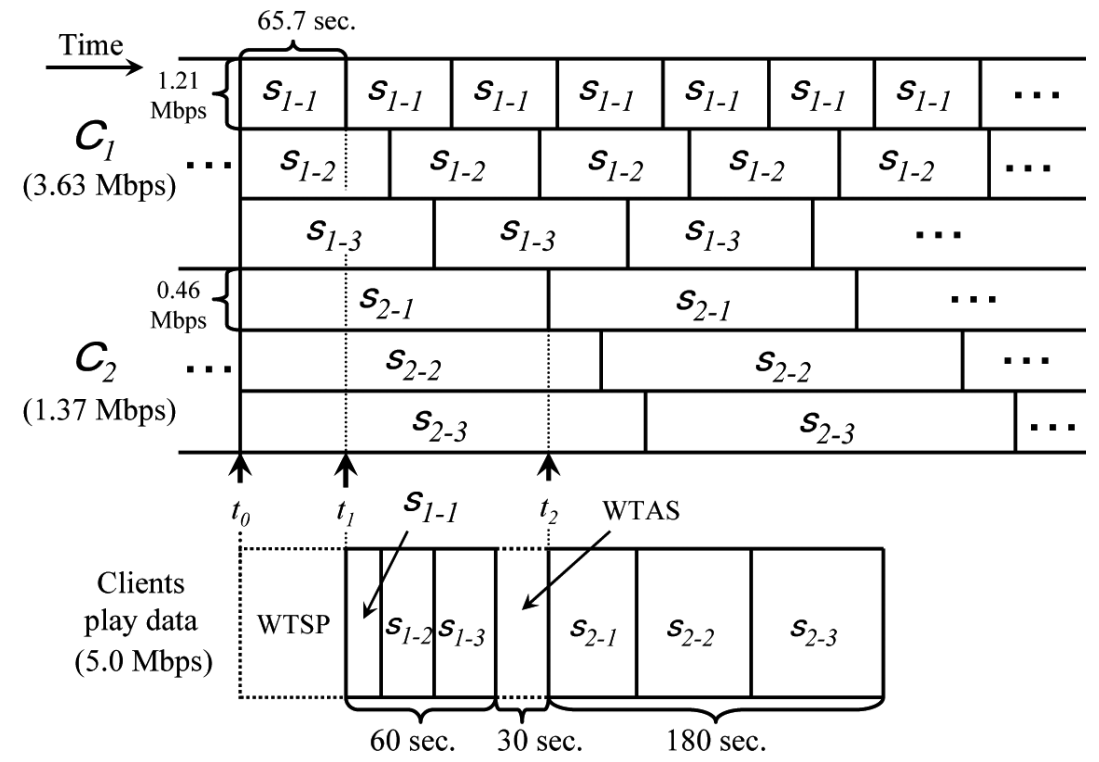

Fig. 5. Example of broadcast schedule under CR-HB method.

This is a naive, but steady approach. We show the average waiting time under different $C_{1}$ in Fig. 4 . The horizontal axis is the bandwidth of $C_{1}$, and the vertical axis is the average waiting time. Total available bandwidth of server is $5.0 \mathrm{Mbps}$, the number of segments is 3 , and the consumption rate is 5.0 Mbps. The playing time of the highlight scene is $60 \mathrm{sec}$, the main program is $180 \mathrm{sec}$, and WTAS is $30 \mathrm{sec}$. In Fig. 4, we can see that the average waiting time is minimum when $C_{1}^{*}=3.63 \mathrm{Mbps}$. In this way, we can find $C_{1}^{*}$. In our evaluation, we use the value of $C_{1}^{*}$ that makes the average waiting time minimum. 


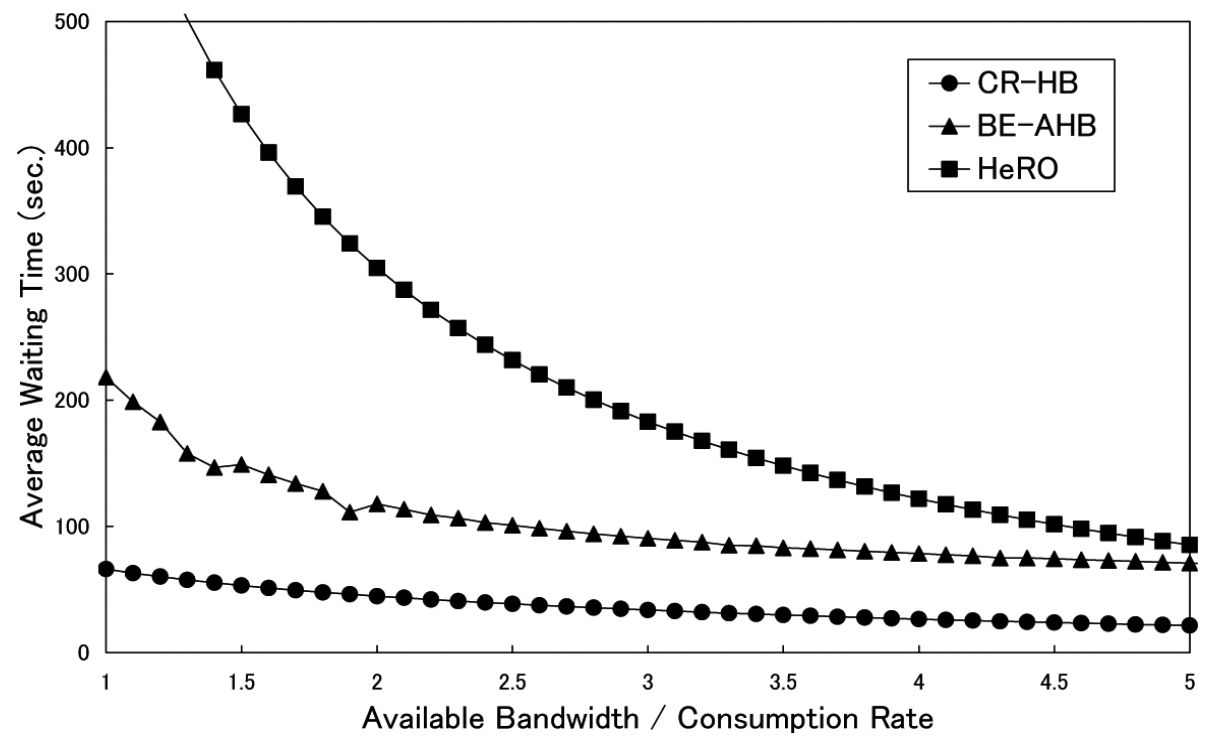

Fig. 6. The average waiting time and the available bandwidth.

\subsection{Implementation}

The server broadcasts segments following the procedure were explained in Subsection 4.4. Continuous media data are partitioned into several segments by the broadcast schedule in the CR-HB method, where the bandwidth of each subchannel is adjusted based on the available bandwidth of the server. In addition, the server broadcasts data repeatedly considering the broadcast schedule.

When the clients require continuous media data from the server, they start to receive them from several broadcast channels. Clients start playing the highlight scene after completely receiving $s_{1-1}$. They receive the data while playing it and store it in their buffer. Clients play $s_{1-2}$ continuously, which is stored in their buffer after finishing the playing of $s_{1-1}$. Clients can play continuous media data without interruption until completely finished receiving it.

For example, a situation that delivers data under the CR-HB method is shown in Fig. 5. The client starts receiving data at $t_{0}$ and starts playing it after receiving $s_{1-1}$ at $t_{1}$. In this case, since the bandwidth of $c_{1-1}$ is $1.21 \mathrm{Mbps}$, the receiving time of $s_{1-1}$ is $65.7 \mathrm{sec}$. Next, when WTAS is $30 \mathrm{sec}$, since the client finishes receiving $s_{2-1}$ at $t_{2}$, it can play $s_{2-1}$ without interruption after finishing WTAS. In Fig. 5, the waiting time under the CR-HB method is reduced $(153.9-66.2) / 153.9 \times 100=57.0 \%$ compared to the BE-AHB method.

\section{Evaluation}

\subsection{Basic idea}

In this section, we evaluate the performance of the CR-HB method with a computational simulation. Actually, there are many network structures for continuous media data broadcasting. However, since the number of patterns is excessive, evaluating the performance of our proposed method for all these patterns was not realistic. Therefore, we used the network configuration shown in Fig. 1 and compared the CR-HB method, the BE-AHB method [15], and the HeRO [12]. 


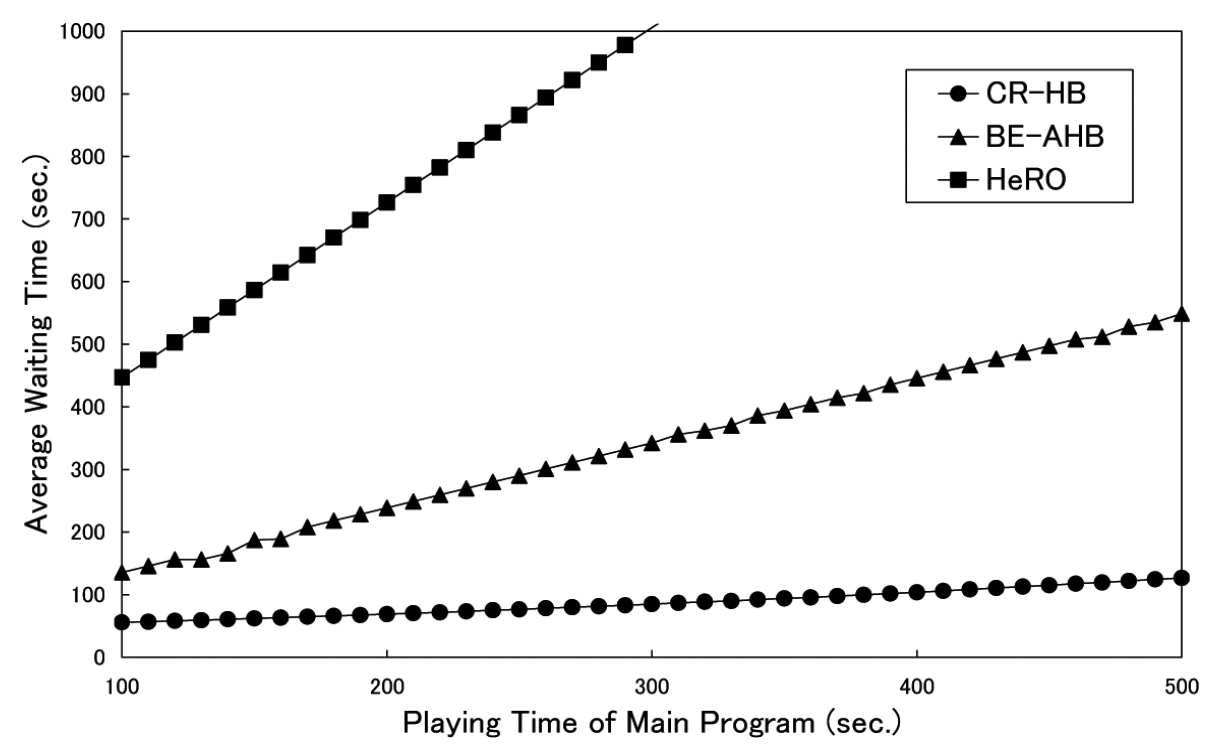

Fig. 7. Average waiting time and playing time of main program.

\subsection{Effect of bandwidth}

Users prefer shorter waiting times. Since the waiting time accepted by users varies, we show that it is reduced with the CR-HB method compared with conventional methods.

The result is shown in Fig. 6. The horizontal axis is the available bandwidth divided by the consumption rate. The vertical axis is the average waiting time. The playing time of the highlight scene is $60 \mathrm{sec}$, that of the main program is $180 \mathrm{sec}$, and WTAS is $30 \mathrm{sec}$. Consumption rate is $5.0 \mathrm{Mbps}$.

In Fig. 6, the average waiting time under the CR-HB method is shorter than the conventional BE-AHB method and HeRO. The CR-HB method schedules segments so that the available bandwidth of $c_{1-1}$ that broadcasts $s_{1-1}$ becomes large. Also, we can effectively reduce the waiting time by scheduling segments considering WTSP. For example, when the available bandwidth is $15 \mathrm{Mbps}$, the waiting time is $33.7 \mathrm{sec}$ under the CR-HB method, $90.5 \mathrm{sec}$ under the BE-AHB method, and $182.9 \mathrm{sec}$ under the HeRO. The average waiting time under the CR-HB method is reduced $62.8 \%$ compared to the BE-AHB method, and $81.6 \%$ compared to the HeRO.

\subsection{Influence of playing time of main program}

Waiting time can be reduced by decreasing the playing time of the highlight scene. However, interruption time may be caused by increasing the playing time of the main program. To evaluate the influence of the playing time of the main program, we calculated the waiting time under different playing times of the main program. The result is shown in Fig. 7. The horizontal axis is the playing time of the main program, and the vertical axis is the average waiting time. The playing time of the highlight scene is 60 $\mathrm{sec}$ and WTAS is $30 \mathrm{sec}$. Consumption rate is $5.0 \mathrm{Mbps}$.

In Fig. 7, when the playing time of the main program increases, the effect of the average waiting time in the CR-HB method is relatively small. The CR-HB method makes the broadcast schedule considering the playing time of the highlight scene and WTAS. On the other hand, the conventional BE-AHB method and HeRO make the broadcast schedule considering both playing time of the highlight scene and the 


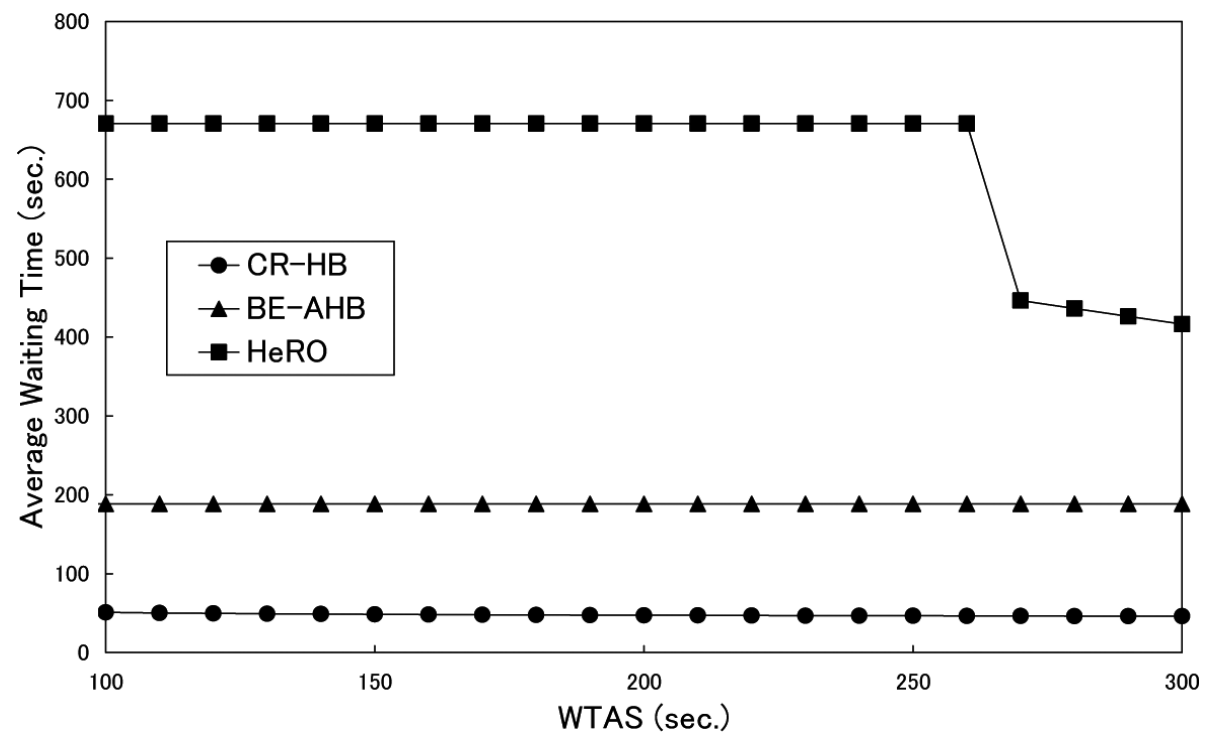

Fig. 8. Average waiting time and waiting time for starting main program.

main program. Therefore, the effect of increasing the playing time of the main program becomes large and waiting time increases.

\subsection{Influence of WTAS}

When we manage a close-range broadcasting service in a public area such as a shopping center or an event hall, there are a lot of users watching content while walking. In these situations, we need to shorten the upper limit of WTAS. On the other hand, when users watch sport in a stadium or standing in a long line, WTAS can be lengthened. To evaluate the influence of WTAS, we calculated the waiting time under different WTAS. The result is shown in Fig. 8. The horizontal axis is WTAS, and the vertical axis is the average waiting time. The available bandwidth is $15 \mathrm{Mbps}$. The playing time of the main program is $180 \mathrm{sec}$, and consumption rate is $5.0 \mathrm{Mbps}$.

In Fig. 8, waiting time under the CR-HB method is shorter than the conventional BE-AHB method and HeRO. In the CR-HB method, waiting time is reduced by making the broadcast schedule considering WTAS. In the conventional BE-AHB method and HeRO, waiting time is not changed significantly when WTAS is between $100 \mathrm{sec}$ and $260 \mathrm{sec}$. These methods make the broadcast schedule considering only waiting time in the receiving time of $s_{1-1}$, and waiting time is not changed by increasing WTAS. However, when WTAS is more than $270 \mathrm{sec}$, waiting time under the HeRO is reduced. In the HeRO, when the available bandwidth for the server has an upper limit, interruption time occurs while playing data.

\section{Conclusion}

In this paper, we proposed a scheduling method to reduce waiting time in close-range broadcasting. In our proposed method, waiting time while playing data is effectively reduced by making the schedule considering the waiting time after the main program. In our evaluations, we showed the available 
bandwidth for which the average waiting time has a minimum value by the computational simulation. Also, we confirmed that the average waiting time has reduced more than the BE-AHB method and the HeRO.

A future direction of this study will involve creating a scheduling method in broadcasting selective contents in which the user watches several content stream while selecting them [18].

\section{Acknowledgment}

This research was supported in part by JSPS Postdoctoral Fellowships for Research Abroad. Also, this work was partially supported by JSPS Grant-in-Aid for Young Scientists (A) numbered 23680007.

\section{References}

[1] WHITE PAPER Information and Communications in Japan (2011). http://www.soumu.go.jp/johotsusintokei/white paper/eng/WP2011/2011-index.html.

[2] Digital TV Broadcasting in Japan, http://www.soumu.go.jp/main_sosiki/joho_tsusin/eng/presentation/pdf/070613_1.pdf.

[3] N. Suzuki and H. Tsujimura, The World's First One-Segment Transmission System Using Weak Radio Waves, Fujitsu, vol.60, no.4, 2009, pp. 341-346.

[4] B. Jinsuk and F.P. Jehan, A Tree-Based Reliable Multicast Scheme Exploiting the Temporal Locality of Transmission Errors, Proc. IEEE Int. Performance, Computing, and Communications Conference (IPCCC 2005), 2005, pp. $275-282$.

[5] L.-S. Juhn and L.M. Tseng, Fast data broadcasting and receiving scheme for popular video service, IEEE Trans Broadcasting 44(1) (1998), 100-105.

[6] J.-F. Paris, S.W. Carter and D.D.E. Long, A hybrid broadcasting protocol for video on demand, Proc. Multimedia Computing and Networking Conference (MMCN '99), 1999, pp. 317-326.

[7] J.-F. Paris, D.D.E. Long and P.E. Mantey, Zero-delay broadcasting protocols for video-on-demand, Proc. ACM Int. Multimedia Conf. (Multimedia ’99), 1999, pp. 189-197.

[8] J.-F. Paris, An Interactive Broadcasting Protocol for Video-on-Demand, Proc. IEEE Int. Performance, Computing, and Communications Conference (IPCCC '01), 2001, pp. 347-353.

[9] S. Viswanathan, and T. Imilelinski, Pyramid broadcasting for video on demand service, Proc. SPIE Multimedia Computing and Networking Conf. (MMCN '95), 1995, pp. 66-77.

[10] L. Shi, P. Sessini, A. Mahanti, Z. Li and D.L. Eager, Scalable Streaming for Heterogeneous Clients, Proc. ACM Multimedia, 2006, pp. 22-27.

[11] Y. Zhao, D.L. Eager and M.K. Vernon, Scalable On-Demand Streaming of Non-Linear Media, Proc. of IEEE INFOCOM, vol.3, 2004, pp. 1522-1533.

[12] K.A. Hua, O. Bagouet and D. Oger, Periodic Broadcast Protocol for Heterogeneous Receivers, Proc. of MMCN, 2003, pp. 220-231.

[13] M. Tantaoui, K. Hua and T. Do, BroadCatch: A Periodic Broadcast Technique for Heterogeneous Video-on-Demand, IEEE Trans Broadcasting 50(3) (2004), 289-301.

[14] R. Janakiraman and M. Waldvogel, Fuzzycast: Efficient Video-on-Demand over Multicast, Proc. IEEE INFOCOM, 2002, 920-929.

[15] T. Yoshihisa, M. Tsukamoto and S. Nishio, A Broadcasting Scheme for Continuous Media Data with Restictions in Data Division, Proc. IPSJ International Conference on Mobile Compuring and Ubiquitous Networking (ICMU'05), 2005, pp. 90-95.

[16] W.-S. Kua and R. Zimmermannb, Nearest neighbor queries with peer-to-peer data sharing in mobile environments, Pervasive and Mobile Computing 4 (2008), 775-788.

[17] Y. Gotoh, K. Suzuki, T. Yoshihisa and M. Kanazawa, A Scheduling Method to Reduce Waiting Time for P2P Streaming Systems, Journal of Mobile Multimedia 5(3) (2009), 255-270.

[18] Y. Gotoh, T. Yoshihisa, M. Kanazawa and Y. Takahashi, A Broadcasting Scheme for Selective Contents Considering Available Bandwidth, IEEE Trans Broadcasting 55(2) (2009), 460-467. 

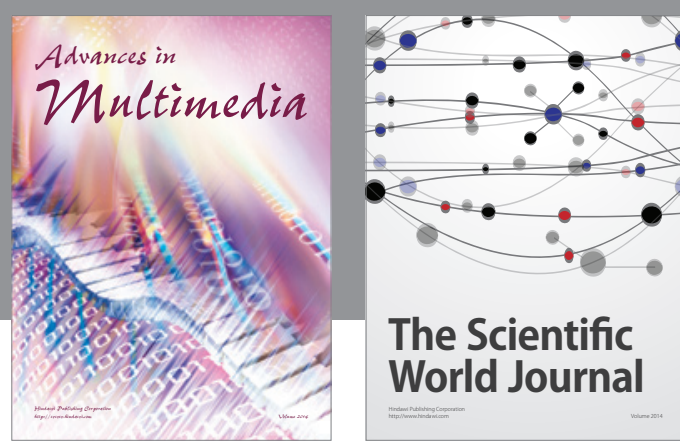

The Scientific World Journal
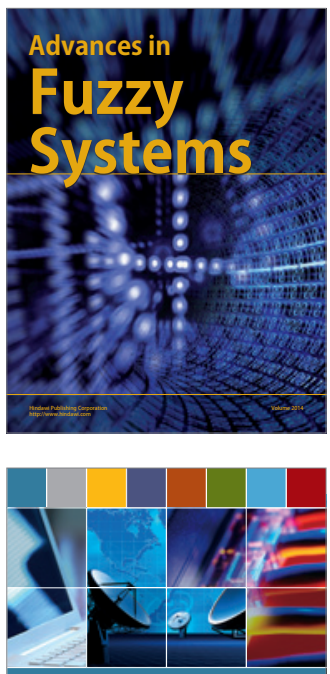

Computer Networks and Communications
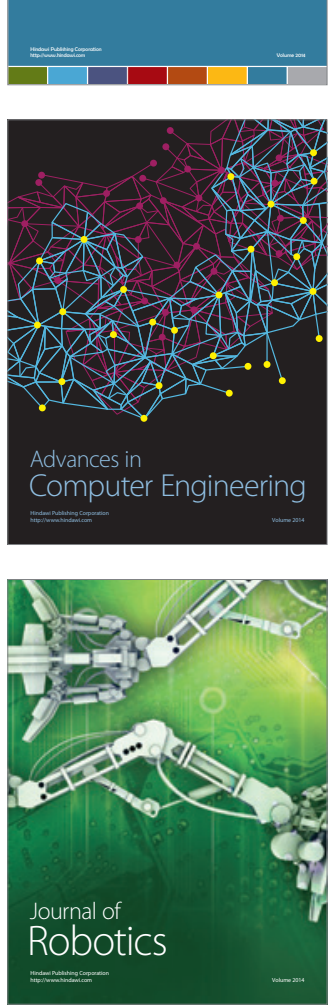
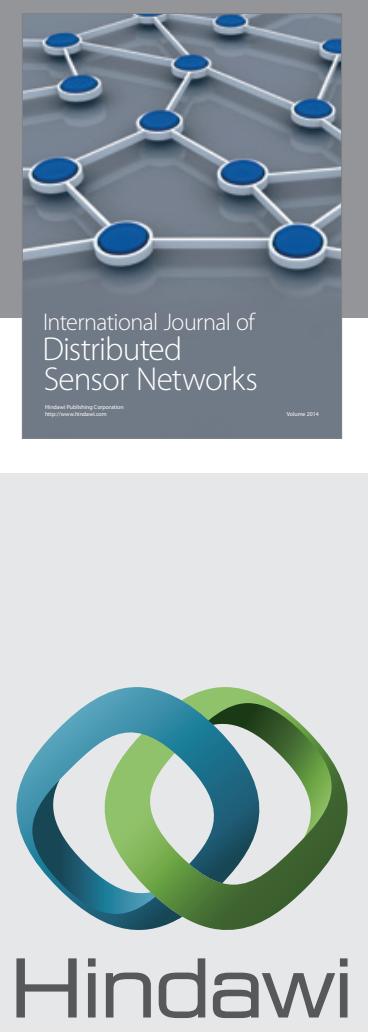

Submit your manuscripts at

http://www.hindawi.com
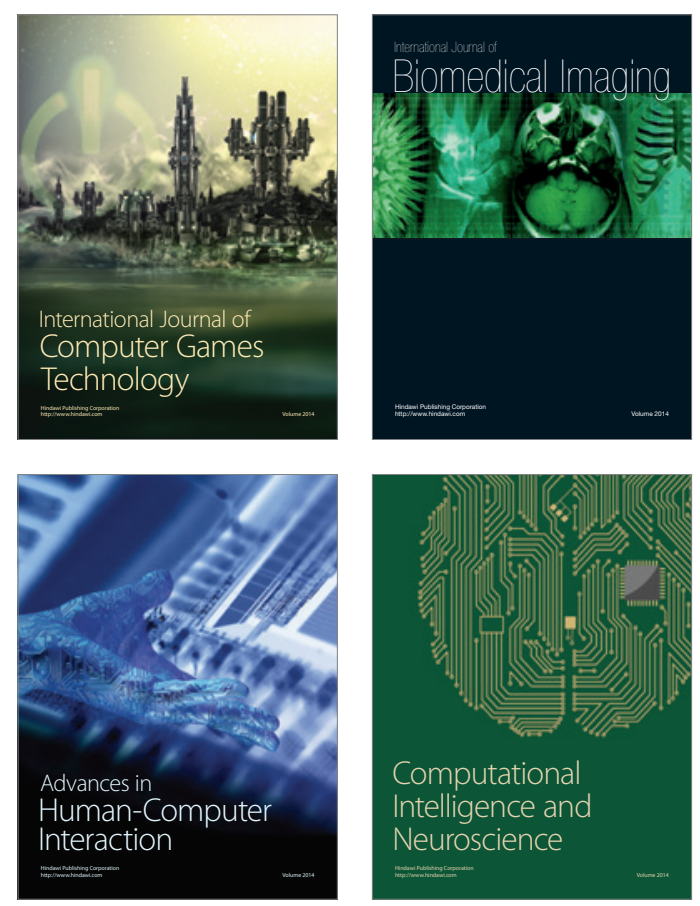
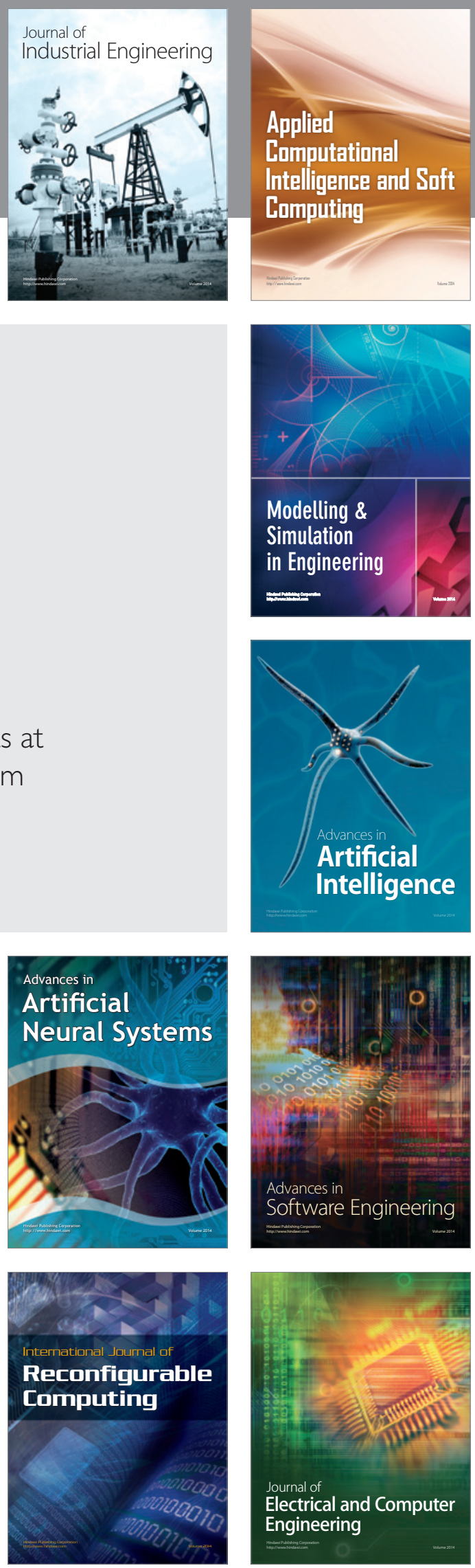